\title{
THE ANALYSIS OF THE ELECTRO-ENCEPHALOGRAM IN CHINESE CHILDREN WITH
} AUTISTIC SPECTRUM DISORDERS

\author{
A.T. Lim $^{1,2}$ \\ ${ }^{\text {I}}$ Pediatrics, Cathay General Hospital, Taipei, ${ }^{2}$ Pediatrics, Cathay General Hopital, Sijhih City, New Taipei, \\ Taiwan R.O.C.
}

Objectives: Autism is classified as global neuro-development disorder especially in behavioral disability. The aim of this study is to evaluate the pattern of electroencephalogram (EEG) in chinese autistic children with or without clinical epilepsy.

Methods: A retrospective chart review of autistic children conducted from outpatient clinics in one medical center from March, 2007 to December, 2010 with cases followed-up at least twice at the same outpatient clinic. The demography of the patients, the standard EEG records, and clinical epilepsy were reviewed.

Results: A total of 139 EEGs of autistic spectrum children were enrolled, age ranging from $2 \mathrm{y} 10 \mathrm{~m}$ to 18 years (means: 7.8years). Male was predominant with the ratio 4:1. Among 139 EEG studies, abnormal EEG was noted in 57 cases. (Male: female 42:15) Twenty-six (46\%) of these 57 cases had multifocal epileptiform discharges, twelve cases (21\%) had localized epileptiform discharges, 4 (7\%) had both multifocal epileptiform discharges with secondary generalized. Localized frontal region epileptiform discharge was found predominantly $(56 \%)$. A higher percentage of epilepsy especially in female population rather than male was found in those with abnormal EEG findings (40\%:29\%).

Conclusions: EEG abnormality only occurs in a certain number of autistic children. The frontal region predominance of EEG discharges associated with beta wave might implicate its major patho-physiological basis in autistic patients. Female autistic children have a clinical significance incidence of underlining seizure activities. 